\title{
Fundamental Kinetics of Supercritical Coal Liquefaction: Effect of Catalysts and Hydrogen-Donor Solvents
}

\author{
Quarterly Report \\ January 1 - March 31, 1997 \\ By \\ Ben J. McCoy; J. M. Smith \\ Giridhar Madras; Yoichi Kodera
}

Work Performed Under Contract No.: DE-FG22-94PC94204

\author{
For \\ U.S. Department of Energy \\ Office of Fossil Energy \\ Federal Energy Technology Center \\ P.O. Box 880 \\ Morgantown, West Virginia 26507-0880
}

By

Department of Chemical Engineering

and Materials Science

University of California

Davis, California 95616 


\section{Disclaimer}

This report was prepared as an account of work sponsored by an agency of the United States Government. Neither the United States Government nor any agency thereof, nor any of their employees, makes any warranty, express or implied, or assumes any legal liability or responsibility for the accuracy, completeness, or usefulness of any information, apparatus, product, or process disclosed, or represents that its use would not infringe privately owned rights. Reference herein to any specific commercial product, process, or service by trade

name, trademark, manufacturer, or otherwise does not necessarily constitute or imply its endorsement, recommendation, or favoring by the United States Government or any agency thereof. The views and opinions of authors expressed herein do not necessarily state or reflect those of the United States Government or any agency thereof. 


\title{
Fundamental Kinetics of Supercritical Coal Liquefaction: Effect of Catalysts and Hydrogen-Donor Solvents
}

\author{
Quarterly Report 10 \\ Reporting Period: January-March, 1997 \\ Principal Investigators: Ben J. McCoy, J.M. Smith \\ Students: Giridhar Madras, Yoichi Kodera \\ April 16, 1997 \\ GRANT NO. DOE DE-FG22-94PC94204--10 \\ Department of Chemical Engineering and Materials Science \\ University of California, Davis, CA 95616. (916) 752-1435
}

PERIOD OF GRANT AWARD: August, 1994 - July, 1998

\section{Disclaimer}

This report was prepared as an account of work sponsored by an agency of the United States Government. Neither the United States Government nor any agency thereof, nor any of their employees, makes any warranty, express or implied, or assumes any legal liability or responsibility for the accuracy, completeness, or usefulness of any information, apparatus, product, or process disclosed or represents that its use would not infringe privately owned rights. Reference herein to any specific commercial product, process or service by trade name, trademark, manufacturer, or otherwise does not necessarily constitute or imply its endorsement, recommendation, or favoring by the United States Government or any agency thereof. The views and opinions of authors expressed herein do not necessarily state or reflect those of the United States Government or any agency thereof.

\section{Table of Contents}

Disclaimer

Executive Summary

Abstract

Introduction

Experiments

Theoretical Model

Results and Discussion

Concluding Remarks

Acknowledgement

References

Figure Captions 


\section{Executive Summary :}

This is the quarterly report on our recent progress toward the overall objective to understand the supercritical fluid extraction of hydrocarbons from coal. Our strategy is to simulate coal as a high molecular-weight polymeric material by studying the degradation of polymers under various conditions. The hypothesis we are testing is that degradation of such macromolecules is applicable to the decomposition (depolymerization) of the coal network.

Polymer degradation and coal liquefaction are influenced strongly by the solvent in the reaction. This motivated our investigation of the effect of hydrogen donor solvents on polymer degradation. In particular, we obtained new experimental data to show how a hydrogen donor, 6-hydroxy tetralin, influences the degradation rate of polystyrene. We also developed a detailed radical mechanism for hydrogen donation based on the Rice-Herzfeld chain reaction concept with the elementary steps of initiation, depropagation, hydrogen abstraction, and termination. Expressions for the degradation rate parameters were obtained by applying continuous distribution kinetics to the MWD of the reacting polymer. The theory explains the different influences of the hydrogen donor solvent on the degradation rate coefficients for different polymers. Though developed for the degradation of polymers, the mechanism and the theory are potentially applicable for chain scission and addition reactions among distributions of paraffins, olefins, and radicals of all chain lengths. The concepts can, in principle, be extended to examine the effect of hydrogen donors on coal liquefaction and on the complex mixture of liquefaction compounds. Based on this work, a research paper titled "Effect of Hydrogen Donors on Polymer Degradation," has been submitted for publication.

Our research paper entitled, "Molecular weight effect on the dynamics of polystyrene degradation," has been accepted for publication by the journal, Industrial and Engineering Chemistry Research. 
The thermal decomposition of hydrocarbon macromolecules, including polymers in solution, is influenced by the presence of hydrogen donors. Depending on the particular polymer, hydrogen donors can increase, decrease, or have no effect on degradation rate. We investigated the concentration effect of the hydrogen donor, 6-hydroxy tetralin, on degradation of polystyrene (2 $\mathrm{g} / \mathrm{L}$ ) dissolved in mineral oil at $275^{\circ} \mathrm{C}$. The time evolution of the molecular weight distribution (MWD) was determined by gel permeation chromatography of samples taken from the batch reactor. The data indicated that the hydrogen donor decreases the polystyrene degradation rate. This is in contrast to the hydrogen donor (tetralin) enhancement of degradation for poly(styrene allyl-alcohol) dissolved in a 1-butanol solution at $150{ }^{\circ} \mathrm{C}$. Because the reaction mechanism for polymer degradation involves radicals, we have developed continuous distribution expressions for polymers and radicals in the elementary reactions by treating molecular weight as a continuous variable. A comprehensive model, which includes expressions for initiationtermination, propagation-depropagation and hydrogen abstraction, accounts for the varied effects of the hydrogen donor on polymer degradation. 
Many processes involve the thermal decomposition of high molecular weight (MW) compounds to a mixture of products. Thermolysis, pyrolysis, coal liquefaction and hydrocarbon cracking, for example, play important roles in the energy and fuels industries. Understanding the thermal decomposition of macromolecules, which involves bond scission and free-radical propagation, is an important aspect of polymer science and engineering (Clough et al., 1996). By pyrolysis, we mean the thermal decomposition of a solid material at high temperatures to yield gas and liquid products of low MW. Thermolysis of polymers in solution produces a mixture of solubilized products. In either case, the decomposition yields a product mixture that often can be described as a continuous function of MW. The time evolution of the molecular weight distribution (MWD) can be examined by continuous distribution kinetics to determine the rate parameters and provide insights into the decomposition mechanisms.

Thermochemical recycling of polymers as either fuel or feedstock has been receiving growing attention in recent years (Miller, 1994). The research has focused on the mechanism of degradation of pure polymers (McCaffrey, 1996). The degradation of polystyrene has been extensively investigated by pyrolysis (Cameron and MacCallum, 1967) though the mechanism and kinetics of polstyrene degradation remain subjects of discussion (McNeill et al., 1990). Degradation of polymers in solution was proposed to counter the problems of low heat transfer rates and high viscosity of the melting polymer commonly encountered in polymer recycling by pyrolysis (Sato et al., 1990). The degradation of polystyrene (Murakata et al., 1993a; Madras et al., 1996), poly(styrene-allyl alcohol) (Wang et al., 1995), poly(methyl methacrylate) (Madras et al., 1996a), poly (p-methyl styrene) (Murakata et al., 1993b) and poly ( $\alpha$-methyl styrene) (Madras et al., 1996b) in solution have been investigated. The continuous distribution approach is appropriate for analysis of solubilized polymer degradation kinetics (McCoy and Madras, 1996) and has also been applied to coal liquefaction (Wang et al., 1993). 
An important parameter in the degradation of the polymers in solution is the influence of the solvent on the reaction. The solvent effect for polystyrene thermal degradation was investigated by Sato et al. (1990). The conversion of polystyrene to low molecular weight products decreased with the increase of the hydrogen donating ability of the solvents. The study, however, did not determine degradation rate coefficients. Madras et al. (1995) found that tetralin enhanced the rate of degradation of poly(styrene-allyl alcohol). Rate coefficients were determined as a function of tetralin concentration and temperature. Madras et al. (1996) found that tetralin had no effect on the degradation of poly( $\alpha$-methyl styrene). These studies indicate the varied effect of the hydrogen donor on polymer decomposition. Though there have been several experimental studies on how hydrogen donor solvents affect the decomposition of hydrocarbons (Song et al., 1994), model compounds typical of coal (Chiba et al., 1991), and coal (Angelova et al., 1989; Chawla et al., 1989; Mochida et al., 1990), an overall theory for the mechanism is not available.

In the current study, we investigate the effect of hydrogen donors on polymer degradation. In particular, new experimental data is presented to show how a hydrogen donor, 6hydroxy tetralin, influences the degradation rate of polystyrene. A detailed radical mechanism is developed based on the Rice-Herzfeld chain reaction concept with the elementary steps of initiation, depropagation, hydrogen abstraction and termination. Expressions for the degradation rate parameters are obtained by applying continuous distribution kinetics to the MWD of the reacting polymer. The theory explains the different influences of the hydrogen donor solvent on the degradation rate coefficients for different polymers. Though developed for the degradation of polymers, the mechanism and the theory are potentially applicable for chain scission and addition reactions among distributions of paraffins, olefins and radicals of all chain lengths. The concepts can, in principle, be extended to examine the effect of hydrogen donors on coal liquefaction and on the complex mixture of liquefaction compounds. 


\section{Experiments}

Analysis of MWDs. The HPLC (Hewlett-Packard 1050) system consists of a $100 \mu \mathrm{L}$ sample loop, a gradient pump, and an on-line variable wavelength ultraviolet (UV) detector. Three PLgel columns (Polymer Lab Inc.) (300 mm x $7.5 \mathrm{~mm}$ ) packed with cross-linked poly(styrene-

divinyl benzene) with pore sizes of 100,500 , and $10^{4} \AA$ are used in series. Tetrahydrofuran (HPLC grade, Fisher Chemicals) was pumped at a constant flow rate of $1.00 \mathrm{~mL} / \mathrm{min}$. Narrow MW polystyrene standards of MW 162 to 0.93 million (Polymer Lab and Aldrich Chemicals) were used to obtain the calibration curve of retention time versus MW.

Polystyrene Degradation. The thermal decomposition of polystyrene in mineral oil was conducted in a $100 \mathrm{~mL}$ flask equipped with a reflux condenser to ensure the condensation and retention of volatiles. $60 \mathrm{~mL}$ of mineral oil (Fisher Chemicals) was heated to $275{ }^{\circ} \mathrm{C}$, and various amounts $(0-0.60 \mathrm{~g})$ of the hydrogen donor, 6-hydroxy tetralin (Aldrich Chemicals), and $0.12 \mathrm{~g}$ of monodisperse polystyrene ( $\mathrm{MW}=110,000)$ (Aldrich Chemicals) were added. The temperature of the solution was measured with a Type $\mathrm{K}$ thermocouple (Fisher Chemicals) and controlled within $\pm 3{ }^{\circ} \mathrm{C}$ using a Thermolyne 45500 power controller. Samples of $1.0 \mathrm{~mL}$ were taken at 15 minute intervals and dissolved in $1.0 \mathrm{~mL}$ of tetrahydrofuran, THF (HPLC grade, Fisher Chemicals). $100 \mu \mathrm{L}$ of this solution was injected into the HPLC-GPC system to obtain the chromatograph, which was converted to MWD with the calibration curve. Because the mineral oil is UV invisible, its MWD was determined by a refractive index (RI) detector. No change in the MWD of mineral oil was observed when the oil was heated for 3 hours at $275{ }^{\circ} \mathrm{C}$ without polystyrene.

\section{Theoretical Model}

According to the Rice-Herzfeld mechanism, polymers can react by transforming their structure without change in MW, e.g. by hydrogen abstraction or isomerization. They can also undergo chain scission to form lower MW products, or undergo addition reactions yielding higher MW products. Chain scission can occur either at the chain-end yielding a specific 
product, or at a random position along the chain yielding a range of lower MW products. The radicals formed by hydrogen abstraction or chain scission are usually influenced by the presence of the hydrogen donor.

Continuous-distribution mass balances are proposed for the various steps involved in the radical mechanism. The rate coefficients are assumed to be independent of MW, a reasonable assumption at low conversions (Madras et al., 1996). The integro-differential equations obtained from the mass balances can be solved in terms of MW moments. In general, the moments are governed by coupled ordinary differential equations that can be solved numerically. In the present treatment, two common assumptions are made that allow the equations to be solved analytically. The long-chain approximation (LCA) (Nigam et al., 1994; Gavalas, 1966) is valid when initiation and termination events are infrequent compared to the hydrogen-abstraction and propagation-depropagation events. Thus the initiation-termination rates are assumed to be negligible. The quasi-stationary state approximation (QSSA) applies when radical concentrations are extremely small and their rates of change are negligible.

\section{Case 1. Reversible random chain scission}

Polymer degradation in some circumstances can occur solely by random chain scission. The following scheme includes the major elementary steps in the Rice-Herzfeld mechanism (Nigam et al., 1994). We represent the chemical species of the reacting polymer, and the radicals as $\mathrm{P}(\mathrm{x})$ and $\mathrm{R}^{\bullet}(\mathrm{x})$ and their MWDs as $\mathrm{p}_{\text {tot }}(\mathrm{x}, \mathrm{t})$ and $\mathrm{r}(\mathrm{x}, \mathrm{t})$, respectively, where $\mathrm{x}$ represents the continuous variable, MW. Since the polymer reactants and random scission products are not distinguished in the continuous distribution model, a single MWD, $\mathrm{p}_{\text {tot }}(\mathrm{x}, \mathrm{t})$, represents the polymer mixture at any time, t. The initiation-termination reactions are represented as

$$
\begin{aligned}
\mathrm{P}(\mathrm{x}) & \stackrel{\mathrm{k}_{\mathrm{f}}}{\Leftrightarrow} \\
& \mathrm{k}_{\mathrm{t}}
\end{aligned}
$$

where $\Leftrightarrow$ represents a reversible reaction. The reversible hydrogen abstraction process is 


$$
\begin{aligned}
\mathrm{P}(\mathrm{x}) & \stackrel{\mathrm{k}_{\mathrm{h}}}{\Leftrightarrow} \mathrm{R}^{\circ}(\mathrm{x}) \\
& \mathrm{k}_{\mathrm{H}}
\end{aligned}
$$

The propagation-depropagation reactions are

$$
\begin{aligned}
& \mathrm{k}_{\mathrm{b}} \\
& \mathrm{R}^{\circ}(\mathrm{x}) \Leftrightarrow \mathrm{P}\left(\mathrm{x}^{\prime}\right)+\mathrm{R}^{\bullet}\left(\mathrm{x}-\mathrm{x}^{\prime}\right) \\
& \mathrm{k}_{\mathrm{a}}
\end{aligned}
$$

The hydrogen donor reactions are

$$
\begin{gathered}
\mathrm{k}_{\mathrm{d}} \\
\mathrm{P}(\mathrm{x})+\mathrm{D} \rightarrow \mathrm{R}^{\circ}(\mathrm{x})+\mathrm{D}^{\prime} \\
\mathrm{k}_{\mathrm{D}} \\
\mathrm{R}^{\bullet}(\mathrm{x})+\mathrm{D} \rightarrow \mathrm{P}(\mathrm{x})+\mathrm{D}^{\prime}
\end{gathered}
$$

D and D' represent the hydrogen donor and its dehydrogenated form, respectively. Because the MW of $\mathrm{P}(\mathrm{x})$ and $\mathrm{R}^{\bullet}(\mathrm{x})$ differ only by the atomic weight of hydrogen, we assume their MWs are the same.

If dissolved hydrogen is present in the solvent, the donor can be regenerated and act as a shuttle to transfer hydrogen atoms to reaction sites,

$$
(1 / 2) \mathrm{H}_{2}+\mathrm{D}^{\prime} \Leftrightarrow \mathrm{D}
$$

Polystyrene degrades rapidly at low reaction times due to the existence of weak links in the polymer main chain (Chiantore et al. (1981); Madras et al. (1996c)). The weak ( $\mathrm{p}_{\mathrm{w}}$ ) and strong (p) links in the polymer $\left(\mathrm{p}_{\text {tot }}\right)$ can be represented by additive distributions,

$$
\mathrm{p}_{\text {tot }}(\mathrm{x}, \mathrm{t})=\mathrm{p}(\mathrm{x}, \mathrm{t})+\mathrm{p}_{\mathrm{w}}(\mathrm{x}, \mathrm{t})
$$

Then the molar concentration of the polymer is the sum of the molar concentrations of the weak and strong links,

$$
\mathrm{p}_{\text {tot }}^{(0)}(\mathrm{t})=\mathrm{p}^{(0)}(\mathrm{t})+\mathrm{p}_{\mathrm{w}}^{(0)}(\mathrm{t})
$$

where $\mathrm{p}_{\text {tot }}{ }^{(0)}$ is the zero moment. The moments, $\mathrm{p}^{(\mathrm{n})}$, are defined as

$$
\mathrm{p}^{(\mathrm{n})}(\mathrm{t})=\int_{0}^{\infty} \mathrm{x}^{\mathrm{n}} \mathrm{p}(\mathrm{x}, \mathrm{t}) \mathrm{dx}
$$


Since the weak link concentration is approximately two orders of magnitude smaller than the strong link concentration (Madras et al., 1996c), only the random rate coefficients of strong links are examined in this study.

The population balance equations for $\mathrm{p}(\mathrm{x}, \mathrm{t})$ and for radical MWD, $\mathrm{r}(\mathrm{x}, \mathrm{t})$, are as follows (Kodera et al., 1997):

$$
\begin{aligned}
& d p / d t=-k_{f} p(x)+k_{t} \int_{0}^{x} r\left(x^{\prime}\right) r\left(x-x^{\prime}\right) d x^{\prime}-k_{h} p(x)+k_{H} r(x)+k_{b} \int_{x}^{\infty} r\left(x^{\prime}\right) \Omega\left(x, x^{\prime}\right) d x^{\prime} \\
& \text { - } k_{a} p(x) \int_{0}^{\infty} r\left(x^{\prime}\right) d x^{\prime}-k_{d} p(x) C+k_{D} r(x) C \\
& \mathrm{dr} / \mathrm{dt}=2 \mathrm{k}_{\mathrm{f}} \int_{\mathrm{x}}^{\infty} \mathrm{p}\left(\mathrm{x}^{\prime}\right) \Omega\left(\mathrm{x}, \mathrm{x}^{\prime}\right) \mathrm{dx^{ \prime }}-\mathrm{k}_{\mathrm{t}} \mathrm{r}(\mathrm{x}) \int_{0}^{\infty} \mathrm{r}\left(\mathrm{x}^{\prime}\right) \mathrm{dx^{ \prime }}+\mathrm{k}_{\mathrm{h}} \mathrm{p}(\mathrm{x})-\mathrm{k}_{\mathrm{H}} \mathrm{r}(\mathrm{x})-\mathrm{k}_{\mathrm{b}} \mathrm{r}(\mathrm{x}) \\
& +\mathrm{k}_{\mathrm{b}} \int_{\mathrm{x}}^{\infty} \mathrm{r}\left(\mathrm{x}^{\prime}\right) \Omega\left(\mathrm{x}, \mathrm{x}^{\prime}\right) \mathrm{dx^{ \prime }}+\mathrm{k}_{\mathrm{a}} \int_{0}^{\mathrm{x}} \mathrm{r}\left(\mathrm{x}-\mathrm{x}^{\prime}\right) \mathrm{p}\left(\mathrm{x}^{\prime}\right) \mathrm{dx^{ \prime }}-\mathrm{k}_{\mathrm{a}} \mathrm{r}(\mathrm{x}) \int_{0}^{\infty} \mathrm{p}\left(\mathrm{x}^{\prime}\right) \mathrm{dx^{ \prime }} \\
& +k_{d} p(x) C-k_{D} r(x) C
\end{aligned}
$$

The initial conditions are $\mathrm{p}(\mathrm{x}, \mathrm{t}=0)=\mathrm{p}_{0}(\mathrm{x})$ and $\mathrm{r}(\mathrm{x}, \mathrm{t})=0$. Applying the moment operation, $\int_{0}^{\infty}[] x^{n} d x$, yields

$$
\begin{aligned}
d p^{(n)} / d t & =-k_{f} p^{(n)}+k_{t} \sum_{j=0}^{n}\left({ }_{j}\right) r^{(j)} r^{(n-j)}-k_{h} p^{(n)}+k_{H} r^{(n)}+k_{b} Z_{n m} r^{(n)}-k_{a} p^{(n)} r^{(0)} \\
& -k_{d} p^{(n)} C+k_{D} r^{(n)} C \\
d r^{(n)} / d t & =2 k_{f} Z_{n m} p^{(n)}-2 k_{t} r^{(n)} r^{(0)}+k_{h} p^{(n)}-k_{H} r^{(n)}-k_{b} r^{(n)}+k_{b} Z_{n m} r^{(n)} \\
& +k_{a} \sum_{j=0}^{n}\left({ }_{j}^{n}\right) r^{(j)} p^{(n-j)}-k_{a} r^{(n)} p^{(0)}+k_{d} p^{(n)} C-k_{D} r^{(n)} C
\end{aligned}
$$

where $\mathrm{Z}_{\mathrm{nm}}=\Gamma(2 \mathrm{~m}+2) \Gamma(\mathrm{m}+\mathrm{n}+1) /[\Gamma(\mathrm{m}+1) \Gamma(2 \mathrm{~m}+\mathrm{n}+2)] \quad($ McCoy and Madras, 1996). The initial conditions for moments are

$$
\mathrm{p}^{(\mathrm{n})}(\mathrm{t}=0)=\mathrm{p}_{0}{ }^{(\mathrm{n})} \text { and } \mathrm{r}^{(\mathrm{n})}(\mathrm{t}=0)=0
$$

Based on the long chain approximation, the rates of initiation and termination are neglected $\left(\mathrm{k}_{\mathrm{a}}=\mathrm{k}_{\mathrm{b}}=0\right)$. Assuming QSSA, we have

$$
\mathrm{dr}(\mathrm{x}, \mathrm{t}) / \mathrm{dt}=0
$$

and thus

$$
\mathrm{dr}^{(\mathrm{n})} / \mathrm{dt}=0
$$

For $\mathrm{n}=0$ in eq. 1.11 , 


$$
\mathrm{r}^{(0)}=\mathrm{kp}^{(0)}
$$

where $\mathrm{k}=\left(\mathrm{k}_{\mathrm{h}}+\mathrm{k}_{\mathrm{d}} \mathrm{C}\right) /\left(\mathrm{k}_{\mathrm{H}}+\mathrm{k}_{\mathrm{D}} \mathrm{C}\right)$ and for $\mathrm{n}=0$, eq. 1.10 becomes

$$
\mathrm{dp}^{(0)} / \mathrm{dt}=\left(\mathrm{k}_{\mathrm{b}}+\mathrm{k}_{\mathrm{a}} \mathrm{p}^{(0)}\right) \mathrm{r}^{(0)}=\left(\mathrm{k}_{\mathrm{b}}+\mathrm{k}_{\mathrm{a}} \mathrm{p}^{(0)}\right) \mathrm{k} \mathrm{p}^{(0)}
$$

Integrating eq. 1.16 with the initial condition,

$$
\mathrm{p}^{(0)}(\mathrm{t}=0)=\mathrm{p}_{0}^{(0)}
$$

yields

$$
\mathrm{p}^{(0)}(\mathrm{t})=1 /\left[\left(\mathrm{k}_{\mathrm{a}} / \mathrm{k}_{\mathrm{b}}\right)+\exp \left(-\mathrm{k} \mathrm{k}_{\mathrm{b}}\right)\left(1 / \mathrm{p}_{0}{ }^{(0)}-\mathrm{k}_{\mathrm{a}} / \mathrm{k}_{\mathrm{b}}\right)\right]
$$

The equilibrium relation is

$$
\mathrm{p}^{(0)}(\mathrm{t} \rightarrow \infty)=\mathrm{k}_{\mathrm{b}} / \mathrm{k}_{\mathrm{a}}
$$

For polystyrene degradation experiments, there was no evidence of higher MW products (Madras et al., 1996) indicating the absence of the addition reaction $\left(\mathrm{k}_{\mathrm{a}}=0\right)$. Thus we have

$$
\mathrm{p}^{(0)}(\mathrm{t})=\mathrm{p}_{0}{ }^{(0)} \exp \left(\mathrm{k}_{\mathrm{b}} \mathrm{kt}\right)
$$

and a plot of $\ln \left(\mathrm{p}^{(0)} / \mathrm{p}_{0}{ }^{(0)}\right)$ is linear in time with slope $\mathrm{k}_{\mathrm{r}}$,

$$
\mathrm{k}_{\mathrm{r}}=\mathrm{k}_{\mathrm{b}}\left(\mathrm{k}_{\mathrm{h}}+\mathrm{k}_{\mathrm{d}} \mathrm{C}\right) /\left(\mathrm{k}_{\mathrm{H}}+\mathrm{k}_{\mathrm{D}} \mathrm{C}\right)
$$

This is the equation showing how the degradation rate coefficient depends on hydrogen donor concentration. Only zeroth moments (molar concentrations) are required to derive this key relationship, but higher moments provide useful auxiliary information.

The summation of first moments $(\mathrm{n}=1)$ for polymer and radicals (total mass concentration) must be constant when there are no losses from the reactor. For $\mathrm{n}=1$, eqs. 1.10 and 1.11 are added to obtain

$$
\mathrm{d}\left[\mathrm{r}^{(1)}+\mathrm{p}^{(1)}\right] / \mathrm{dt}=0
$$

confirming the mass balance. Applying QSSA,

$$
\mathrm{dr}^{(1)} / \mathrm{dt}=0
$$

we have

$$
\mathrm{dp}^{(1)} / \mathrm{dt}=0
$$

which is integrated with the initial condition (eq. 1.12 with $n=1$ ), 


$$
\mathrm{p}^{(1)}(\mathrm{t})=\mathrm{p}_{0}^{(1)}
$$

The radical mass concentration (eq. 1.11) becomes

$$
\mathrm{r}^{(1)}=\left(\mathrm{k}_{\mathrm{h}}+\mathrm{k}_{\mathrm{d}} \mathrm{C}+\mathrm{k}_{\mathrm{a}} \mathrm{r}^{(0)}\right) \mathrm{p}_{0}^{(1)} /\left(\mathrm{k}_{\mathrm{H}}+\mathrm{k}_{\mathrm{D}} \mathrm{C}+\mathrm{k}_{\mathrm{b}} / 2\right)
$$

The second moment equations $(\mathrm{n}=2)$ are

$$
\begin{aligned}
\mathrm{dp}^{(2)} / \mathrm{dt} & =-\left(\mathrm{k}_{\mathrm{h}}+\mathrm{k}_{\mathrm{d}} \mathrm{C}\right) \mathrm{p}^{(2)}+\left(\mathrm{k}_{\mathrm{H}}+\mathrm{k}_{\mathrm{D}} \mathrm{C}\right) \mathrm{r}^{(2)}+\left(\mathrm{k}_{\mathrm{b}} / 3\right) \mathrm{r}^{(2)}-\mathrm{k}_{\mathrm{a}} \mathrm{p}^{(2)} \mathrm{r}^{(0)} \\
\mathrm{dr} \mathrm{r}^{(2)} / \mathrm{dt} & =\left(\mathrm{k}_{\mathrm{h}}+\mathrm{k}_{\mathrm{d}} \mathrm{C}\right) \mathrm{p}^{(2)}-\left(\mathrm{k}_{\mathrm{H}^{+}}+\mathrm{k}_{\mathrm{D}} \mathrm{C}\right) \mathrm{r}^{(2)}-\left(2 \mathrm{k}_{\mathrm{b}} / 3\right) \mathrm{r}^{(2)} \\
+ & \mathrm{k}_{\mathrm{a}}\left(\mathrm{p}^{(2)} \mathrm{r}^{(0)}+2 \mathrm{p}^{(1)} \mathrm{r}^{(1)}\right)
\end{aligned}
$$

Since $\mathrm{dr}^{(2)} / \mathrm{dt}=0$, then

$$
\mathrm{dp}^{(2)} / \mathrm{dt}=-\left(\mathrm{k}_{\mathrm{b}} / 3\right) \mathrm{r}^{(2)}+2 \mathrm{k}_{\mathrm{a}} \mathrm{p}^{(1)} \mathrm{r}^{(1)}
$$

From Eq. 1.28,

$$
\mathrm{r}^{(2)}=\left[\left(\mathrm{k}_{\mathrm{h}}+\mathrm{k}_{\mathrm{d}} \mathrm{C}+\mathrm{k}_{\mathrm{a}} \mathrm{r}^{(0)}\right) \mathrm{p}^{(2)}+2 \mathrm{k}_{\mathrm{a}} \mathrm{p}^{(1)} \mathrm{r}^{(1)}\right] /\left(\mathrm{k}_{\mathrm{H}}+\mathrm{k}_{\mathrm{D}} \mathrm{C}+2 \mathrm{k}_{\mathrm{b}} / 3\right)
$$

Substitution of $\mathrm{p}^{(1)}, \mathrm{r}^{(1)}$, and $\mathrm{r}^{(2)}$ in eq. 1.29 and integration with the initial condition yield,

$$
\begin{aligned}
\mathrm{p}^{(2)}(\mathrm{t})= & {\left[4 \mathrm{k}_{\mathrm{a}}\left(\mathrm{k}_{\mathrm{b}}+3\left(\mathrm{k}_{\mathrm{H}}+\mathrm{k}_{\mathrm{D}} \mathrm{C}\right)\right) \mathrm{p}_{0}^{(1)^{2}} / \mathrm{k}_{\mathrm{b}}\left(\mathrm{k}_{\mathrm{b}}+2\left(\mathrm{k}_{\mathrm{H}}+\mathrm{k}_{\mathrm{D}} \mathrm{C}\right)\right)\right] } \\
& +\left[\mathrm{p}_{\mathrm{o}}^{(2)}-4 \mathrm{k}_{\mathrm{a}}\left(\mathrm{k}_{\mathrm{b}}+3\left(\mathrm{k}_{\mathrm{H}}+\mathrm{k}_{\mathrm{D}} \mathrm{C}\right)\right) \mathrm{p}_{0}^{(1)^{2}} /\left(\mathrm{k}_{\mathrm{b}}\left(\mathrm{k}_{\mathrm{b}}+2\left(\mathrm{k}_{\mathrm{H}}+\mathrm{k}_{\mathrm{D}} \mathrm{C}\right)\right)\right)\right] \\
& \exp \left[\mathrm{k}_{\mathrm{b}}\left(\mathrm{k}_{\mathrm{h}} \mathrm{t}+\log \mathrm{k}_{\mathrm{b}}-\log \left(\mathrm{k}_{\mathrm{b}}-\mathrm{k}_{\mathrm{a}} \mathrm{p}_{0}^{(0)}\left(1-\exp \left(\mathrm{k}_{\mathrm{a}} \mathrm{k} \mathrm{t}\right)\right)-\log \mathrm{k}_{\mathrm{b}}\right)\right)\right. \\
& \left./\left(2 \mathrm{k}_{\mathrm{b}}+3\left(\mathrm{k}_{\mathrm{H}}+\mathrm{k}_{\mathrm{D}} \mathrm{C}\right)\right)\right]
\end{aligned}
$$

In the absence of addition $\left(\mathrm{k}_{\mathrm{a}}=0\right)$,

$$
\mathrm{p}^{(2)}(\mathrm{t})=\mathrm{p}_{0}^{(2)} \exp \left[-\mathrm{k}_{\mathrm{b}}\left(\mathrm{k}_{\mathrm{h}}+\mathrm{k}_{\mathrm{d}} \mathrm{C}\right) \mathrm{t} /\left(3\left(\mathrm{k}_{\mathrm{H}}+\mathrm{k}_{\mathrm{D}} \mathrm{C}\right)+2 \mathrm{k}_{\mathrm{b}}\right)\right]
$$

which is identical to the $\mathrm{p}^{(2)}(\mathrm{t})$ expression derived by a simpler model (McCoy and Madras, 1996) when $2 \mathrm{k}_{\mathrm{b}}<<3\left(\mathrm{k}_{\mathrm{H}}+\mathrm{k}_{\mathrm{D}}\right.$ C), i.e.,

$$
\mathrm{p}^{(2)}(\mathrm{t}) \simeq \mathrm{p}_{0}^{(2)} \exp \left(-\mathrm{k}_{\mathrm{r}} \mathrm{t} / 3\right)
$$

Thus a plot of $\ln \left(\mathrm{p}^{(2)} / \mathrm{p}_{0}{ }^{(2)}\right)$ would be linear in time with slope $\mathrm{k}_{\mathrm{r}} / 3$, as found by McCoy and Madras (1996).

\section{Case 2. Reversible Chain-End Scission}


Polymers like poly( $\alpha$-methyl styrene) undergo degradation by chain-end scission yielding monomers and other low-MW specific products, $\mathrm{Q}_{\mathrm{S}}\left(\mathrm{x}_{\mathrm{S}}\right)$. The formation of chain-end radicals by a reversible random-scission initiation/termination reaction is

$$
\mathrm{P}(\mathrm{x}) \underset{\mathrm{k}_{\mathrm{ts}}}{\Leftrightarrow} \mathrm{R}_{\mathrm{e}} \cdot(\mathrm{x})+\mathrm{R}_{\mathrm{e}} \cdot\left(\mathrm{x}-\mathrm{x}^{\prime}\right)
$$

Hydrogen abstraction by the chain-end radical is considered reversible, $\mathrm{k}_{\mathrm{he}}$

$$
\mathrm{P}(\mathrm{x}) \underset{\mathrm{kHe}^{\mathrm{He}}}{\Leftrightarrow} \mathrm{R}_{\mathrm{e}}{ }^{\circ}(\mathrm{x})
$$

The chain-end radical, $\mathrm{R}_{\mathrm{e}}{ }^{\bullet}(\mathrm{x})$, can also undergo radical isomerization to form a specific radical, $\mathrm{R}_{\mathrm{S}} \cdot(\mathrm{x})$, via a cyclic transition state,

$$
\begin{aligned}
& \mathrm{k}_{\mathrm{ih}} \\
& \mathrm{R}_{\mathrm{e}}{ }^{\cdot}(\mathrm{x}) \Leftrightarrow \mathrm{R}_{\mathrm{s}}{ }^{\cdot}(\mathrm{x}) \\
& \mathrm{k}_{\mathrm{iH}}
\end{aligned}
$$

The reversible, propagation-depropagation reactions whereby a specific radical yields a specific product and a chain-end radical is

$$
\begin{aligned}
& \mathrm{k}_{\mathrm{bs}} \\
& \mathrm{R}_{\mathrm{S}}{ }^{\cdot}(\mathrm{x}) \Leftrightarrow \mathrm{Q}_{\mathrm{s}}\left(\mathrm{x}_{\mathrm{S}}\right)+\mathrm{R}_{\mathrm{e}}{ }^{\cdot}\left(\mathrm{x}-\mathrm{x}_{\mathrm{S}}\right) \\
& \mathrm{k}_{\mathrm{as}}
\end{aligned}
$$

The reactions of the hydrogen donor expressed in terms of D, the hydrogenated and D', the dehydrogenated forms of the donor, are

$$
\begin{gathered}
\mathrm{P}(\mathrm{x})+\mathrm{D} \underset{\mathrm{k}_{\mathrm{De}}}{\rightarrow} \mathrm{R}_{\mathrm{e}}{ }^{\circ}(\mathrm{x})+\mathrm{D}^{\prime} \\
\mathrm{R}_{\mathrm{e}} \cdot(\mathrm{x})+\mathrm{D} \rightarrow \mathrm{P}(\mathrm{x})+\mathrm{D}^{\prime}
\end{gathered}
$$

Based on the long-chain approximation, eq. 2.1 can be neglected. The mechanism represented by Eqs. 2.2 - 2.4 describes the role of the end radical. $\mathrm{Q}_{\mathrm{s}}$ represents the specific compound of $\mathrm{MW} \mathrm{x}_{\mathrm{S}}$ formed due to the chain-end scission of the polymer with strong links, $\mathrm{P}(\mathrm{x})$.

The balance equations for $\mathrm{p}(\mathrm{x}, \mathrm{t}), \mathrm{q}_{\mathrm{s}}(\mathrm{x}, \mathrm{t}), \mathrm{r}_{\mathrm{e}}(\mathrm{x}, \mathrm{t})$, and $\mathrm{r}_{\mathrm{s}}(\mathrm{x}, \mathrm{t})$ are

$$
\begin{aligned}
& \mathrm{dp} / \mathrm{dt}=-\left(\mathrm{k}_{\mathrm{he}}+\mathrm{k}_{\mathrm{de}} \mathrm{C}\right) \mathrm{p}(\mathrm{x})+\left(\mathrm{k}_{\mathrm{He}}+\mathrm{k}_{\mathrm{De}} \mathrm{C}\right) \mathrm{r}_{\mathrm{e}}(\mathrm{x}) \\
& \mathrm{dq}_{\mathrm{s}} / \mathrm{dt}=\mathrm{k}_{\mathrm{bs}} \int_{\mathrm{x}}^{\infty} \mathrm{r}_{\mathrm{s}}\left(\mathrm{x}^{\prime}\right) \delta\left(\mathrm{x}^{\prime}-\mathrm{x}_{\mathrm{S}}\right) \mathrm{dx} \mathrm{x}^{\prime}-\mathrm{k}_{\mathrm{as}} \mathrm{q}_{\mathrm{s}}(\mathrm{x}) \int_{0}^{\infty} \mathrm{r}_{\mathrm{e}}\left(\mathrm{x}^{\prime}\right) \mathrm{dx} \mathrm{x}^{\prime}
\end{aligned}
$$


94204R10.PDF

$$
\begin{aligned}
\mathrm{dr}_{\mathrm{e}} / \mathrm{dt}= & \left(\mathrm{k}_{\mathrm{he}}+\mathrm{k}_{\mathrm{de}} \mathrm{C}\right) \mathrm{p}(\mathrm{x})-\left(\mathrm{k}_{\mathrm{He}}+\mathrm{k}_{\mathrm{De}} \mathrm{C}\right) \mathrm{r}_{\mathrm{e}}(\mathrm{x})-\mathrm{k}_{\mathrm{ih}} \mathrm{r}_{\mathrm{e}}(\mathrm{x})+\mathrm{k}_{\mathrm{iH}} \mathrm{r}_{\mathrm{s}}(\mathrm{x}) \\
& +\mathrm{k}_{\mathrm{bs}} \int_{\mathrm{x}}^{\infty} \mathrm{r}_{\mathrm{s}}\left(\mathrm{x}^{\prime}\right) \delta\left[\mathrm{x}-\left(\mathrm{x}^{\prime}-\mathrm{x}_{\mathrm{s}}\right)\right] \mathrm{dx^{ \prime }}-\mathrm{k}_{\mathrm{as}} \mathrm{r}_{\mathrm{e}}(\mathrm{x}) \int_{0}^{\infty} \mathrm{q}_{\mathrm{s}}\left(\mathrm{x}^{\prime}\right) \mathrm{dx}^{\prime} \\
\mathrm{dr}_{\mathrm{s}} / \mathrm{dt}= & \mathrm{k}_{\mathrm{ih}} \mathrm{r}_{\mathrm{e}}(\mathrm{x})-\mathrm{k}_{\mathrm{bs}} \mathrm{r}_{\mathrm{s}}(\mathrm{x})-\mathrm{k}_{\mathrm{iH}} \mathrm{r}_{\mathrm{s}}(\mathrm{x})+\mathrm{k}_{\mathrm{as}} \int_{0}^{\mathrm{x}} \mathrm{r}_{\mathrm{e}}\left(\mathrm{x}^{\prime}-\mathrm{x}_{\mathrm{s}}\right) \mathrm{q}_{\mathrm{s}}\left(\mathrm{x}_{\mathrm{s}}\right) \mathrm{dx^{ \prime }}
\end{aligned}
$$

The initial conditions are

$$
\mathrm{p}(\mathrm{x}, \mathrm{t}=0)=\mathrm{p}_{0}(\mathrm{x}) \quad \text { and } \quad \mathrm{q}_{\mathrm{s}}(\mathrm{x}, \mathrm{t}=0)=\mathrm{r}_{\mathrm{e}}(\mathrm{x}, \mathrm{t}=0)=\mathrm{r}_{\mathrm{s}}(\mathrm{x}, \mathrm{t}=0)=0
$$

Applying the moment operation to each balance equation yields

$$
\begin{aligned}
& d p^{(n)} / d t=-\left(k_{h e}+k_{d e} C\right) p^{(n)}+\left(k_{H e}+k_{D e} C\right) r_{e}{ }^{(n)} \\
& \mathrm{dq}_{\mathrm{s}}{ }^{(\mathrm{n})} / \mathrm{dt}=\mathrm{k}_{\mathrm{bs}} \mathrm{x}_{\mathrm{s}}{ }^{\mathrm{n}} \mathrm{r}_{\mathrm{s}}{ }^{(0)}-\mathrm{k}_{\mathrm{as}} \mathrm{q}_{\mathrm{s}}{ }^{(\mathrm{n})} \mathrm{r}_{\mathrm{e}}{ }^{(0)} \\
& \mathrm{dr}_{\mathrm{e}}^{(\mathrm{n})} / \mathrm{dt}=\left(\mathrm{k}_{\mathrm{he}}+\mathrm{k}_{\mathrm{de}} \mathrm{C}\right) \mathrm{p}^{(\mathrm{n})}-\left(\mathrm{k}_{\mathrm{He}}+\mathrm{k}_{\mathrm{De}} \mathrm{C}\right) \mathrm{r}_{\mathrm{e}}{ }^{(\mathrm{n})}-\mathrm{k}_{\mathrm{ih}} \mathrm{r}_{\mathrm{e}}^{(\mathrm{n})}-\mathrm{k}_{\mathrm{iH}} \mathrm{r}_{\mathrm{e}}^{(\mathrm{n})}-\mathrm{k}_{\mathrm{as}} \mathrm{r}_{\mathrm{e}}{ }^{(\mathrm{n})} \mathrm{p}_{\mathrm{s}}{ }^{(0)} \\
& +k_{b s} \sum_{j=0}^{n}\left({ }_{j}^{n}\right)\left(x_{s}\right)^{j}(-1)^{j} r_{s}^{(n-j)} \\
& \mathrm{dr}_{\mathrm{s}}^{(\mathrm{n})} / \mathrm{dt}=\mathrm{k}_{\mathrm{ih}} \mathrm{r}_{\mathrm{e}}^{(\mathrm{n})}-\mathrm{k}_{\mathrm{bs}} \mathrm{r}_{\mathrm{s}}^{(\mathrm{n})}-\mathrm{k}_{\mathrm{iH}} \mathrm{r}_{\mathrm{s}}^{(\mathrm{n})}+\mathrm{k}_{\mathrm{as}} \sum_{j=0}^{\mathrm{n}}\left({ }_{\mathrm{j}}^{\mathrm{n}}\right) \mathrm{r}_{\mathrm{e}}^{(\mathrm{j})} \mathrm{q}_{\mathrm{s}}^{(\mathrm{n}-\mathrm{j})}
\end{aligned}
$$

The initial conditions for moments are

$$
\mathrm{p}^{(\mathrm{n})}(\mathrm{t}=0)=\mathrm{p}_{0}{ }^{(\mathrm{n})} \quad \text { and } \quad \mathrm{q}_{\mathrm{s}}^{(\mathrm{n})}(\mathrm{t}=0)=\mathrm{r}_{\mathrm{e}}^{(\mathrm{n})}(\mathrm{t}=0)=\mathrm{r}_{\mathrm{s}}^{(\mathrm{n})}(\mathrm{t}=0)=0(2.14)
$$

Similar to the treatment in random chain scission, zeroth moments are governed by the following differential equations, where the QSSA for radical species has been applied:

$$
\begin{aligned}
\mathrm{dp}^{(0)} / \mathrm{dt} & =-\left(\mathrm{k}_{\mathrm{he}}+\mathrm{k}_{\mathrm{de}} \mathrm{C}\right) \mathrm{p}^{(0)}+\left(\mathrm{k}_{\mathrm{He}}+\mathrm{k}_{\mathrm{De}} \mathrm{C}\right) \mathrm{r}_{\mathrm{e}}^{(0)} \\
\mathrm{dq}_{\mathrm{s}}{ }^{(0)} / \mathrm{dt} & =\mathrm{k}_{\mathrm{bs}} \mathrm{r}_{\mathrm{s}}^{(0)}-\mathrm{k}_{\mathrm{as}} \mathrm{q}_{\mathrm{s}}^{(0)} \mathrm{r}_{\mathrm{e}}{ }^{(0)} \\
\mathrm{dr} \mathrm{e}^{(0)} / \mathrm{dt} & =\left(\mathrm{k}_{\mathrm{he}}+\mathrm{k}_{\mathrm{de}} \mathrm{C}\right) \mathrm{p}^{(0)}-\left(\mathrm{k}_{\mathrm{He}}+\mathrm{k}_{\mathrm{De}} \mathrm{C}\right) \mathrm{r}_{\mathrm{e}}^{(0)}-\mathrm{k}_{\mathrm{ih}} \mathrm{r}_{\mathrm{e}}^{(0)} \\
& +\mathrm{k}_{\mathrm{bs}} \mathrm{r}_{\mathrm{s}}^{(0)}-\mathrm{k}_{\mathrm{as}} \mathrm{r}_{\mathrm{e}}^{(0)} \mathrm{q}_{\mathrm{s}}^{(0)}=0 \\
\mathrm{dr}_{\mathrm{s}}{ }^{(0)} / \mathrm{dt} & =\mathrm{k}_{\mathrm{ih}} \mathrm{r}_{\mathrm{e}}^{(0)}-\mathrm{k}_{\mathrm{bs}} \mathrm{r}_{\mathrm{s}}^{(0)}+\mathrm{k}_{\mathrm{as}} \mathrm{r}_{\mathrm{e}}^{(0)} \mathrm{q}_{\mathrm{s}}^{(0)}=0
\end{aligned}
$$

Summing eqs. 2.17 and 2.18 yields

$$
\mathrm{r}_{\mathrm{e}}^{(0)}=\mathrm{k}_{\mathrm{e}} \mathrm{p}^{(0)}
$$

where $\mathrm{k}_{\mathrm{e}}=\left(\mathrm{k}_{\mathrm{he}}+\mathrm{k}_{\mathrm{de}} \mathrm{C}\right) /\left(\mathrm{k}_{\mathrm{He}}+\mathrm{k}_{\mathrm{De}} \mathrm{C}\right)$. Then, eq. 2.15 is

$$
\mathrm{dp}^{(0)} / \mathrm{dt}=0
$$

which can be solved with the initial condition, eq. 2.14 , and the solution is 


$$
\mathrm{p}^{(0)}(\mathrm{t})=\mathrm{p}_{0}^{(0)}
$$

This indicates that the molar concentration of polymer is unchanged by chain-end scission. Eq. 2.18 gives

$$
\mathrm{r}_{\mathrm{s}}^{(0)}=\mathrm{r}_{\mathrm{e}}^{(0)}\left(\mathrm{k}_{\mathrm{ih}}+\mathrm{k}_{\mathrm{as}} \mathrm{q}_{\mathrm{s}}^{(0)}\right) /\left(\mathrm{k}_{\mathrm{bs}}+\mathrm{k}_{\mathrm{iH}}\right)
$$

When the addition reaction is insignificant, then $\mathrm{k}_{\mathrm{as}}=0$ and eq. 2.16 can be solved with eqs. 2.19 and 2.22 ,

$$
\mathrm{q}_{\mathrm{s}}^{(0)}(\mathrm{t})=\mathrm{k}_{\mathrm{s}} \mathrm{p}_{0}{ }^{(0)} \mathrm{t}
$$

where

$$
\begin{aligned}
\mathrm{k}_{\mathrm{s}} & =\mathrm{k}_{\mathrm{e}} \mathrm{k}_{\mathrm{bs}} \mathrm{k}_{\mathrm{ih}} /\left(\mathrm{k}_{\mathrm{bs}}+\mathrm{k}_{\mathrm{iH}}\right) \\
& =\left(\mathrm{k}_{\mathrm{he}}+\mathrm{k}_{\mathrm{de}} \mathrm{C}\right) \mathrm{k}_{\mathrm{bs}} \mathrm{k}_{\mathrm{ih}} /\left(\left(\mathrm{k}_{\mathrm{bs}}+\mathrm{k}_{\mathrm{iH}}\right)\left(\mathrm{k}_{\mathrm{He}}+\mathrm{k}_{\mathrm{De}} \mathrm{C}\right)\right)
\end{aligned}
$$

This is a key result for chain-end scission influenced by hydrogen donor concentration, $\mathrm{C}$, and similar to eq. 1.21 for random chain scission. A plot of $\mathrm{q}_{\mathrm{s}}{ }^{(0)}(\mathrm{t}) / \mathrm{p}_{0}{ }^{(0)}$ versus time would be linear with a slope $\mathrm{k}_{\mathrm{s}}$, which depends on $\mathrm{C}$. This behavior has been experimental observed for the chain-end scission of poly(styrene-allyl alcohol) (Madras et al., 1995).

First moment equations for each species are

$$
\begin{aligned}
& \mathrm{dp}^{(1)} / \mathrm{dt}=-\left(\mathrm{k}_{\mathrm{he}}+\mathrm{k}_{\mathrm{de}} \mathrm{C}\right) \mathrm{p}^{(1)}+\left(\mathrm{k}_{\mathrm{He}}+\mathrm{k}_{\mathrm{De}} \mathrm{C}\right) \mathrm{r}_{\mathrm{e}}{ }^{(1)} \\
& \mathrm{dq}_{\mathrm{s}}^{(1)} / \mathrm{dt}=\mathrm{k}_{\mathrm{bs}} \mathrm{x}_{\mathrm{s}} \mathrm{r}_{\mathrm{s}}^{(0)}-\mathrm{k}_{\mathrm{as}} \mathrm{q}_{\mathrm{s}}^{(1)} \mathrm{r}_{\mathrm{e}}{ }^{(0)} \\
& \mathrm{dr}_{\mathrm{e}}{ }^{(1)} / \mathrm{dt}=\left(\mathrm{k}_{\mathrm{he}}+\mathrm{k}_{\mathrm{de}} \mathrm{C}\right) \mathrm{p}^{(1)}-\left(\mathrm{k}_{\mathrm{He}}+\mathrm{k}_{\mathrm{De}} \mathrm{C}\right) \mathrm{r}_{\mathrm{e}}^{(1)}-\mathrm{k}_{\mathrm{ih}} \mathrm{r}_{\mathrm{e}}^{(1)} \\
& \quad+\mathrm{k}_{\mathrm{bs}}\left(\mathrm{r}_{\mathrm{s}}{ }^{(1)}-\mathrm{x}_{\mathrm{s}} \mathrm{r}_{\mathrm{s}}^{(0)}\right)-\mathrm{k}_{\mathrm{as}} \mathrm{r}_{\mathrm{e}}^{(1)} \mathrm{q}_{\mathrm{s}}^{(0)} \\
& \mathrm{dr}_{\mathrm{s}}{ }^{(1)} / \mathrm{dt}=\mathrm{k}_{\mathrm{ih}} \mathrm{r}_{\mathrm{e}}^{(1)}-\mathrm{k}_{\mathrm{bs}} \mathrm{r}_{\mathrm{s}}^{(1)}+\mathrm{k}_{\mathrm{as}}\left(\mathrm{r}_{\mathrm{e}}^{(1)} \mathrm{q}_{\mathrm{s}}^{(0)}+\mathrm{r}_{\mathrm{e}}^{(0)} \mathrm{q}_{\mathrm{s}}^{(1)}\right)
\end{aligned}
$$

The summation of first moments for polymer and radicals yields

$$
\mathrm{d}\left[\mathrm{p}^{(1)}+\mathrm{q}_{\mathrm{s}}^{(1)}+\mathrm{r}_{\mathrm{e}}^{(1)}+\mathrm{r}_{\mathrm{s}}^{(1)}\right] / \mathrm{dt}=0
$$

Since $\mathrm{dr}_{\mathrm{e}}{ }^{(1)} / \mathrm{dt}=\mathrm{dr}_{\mathrm{s}}{ }^{(1)} / \mathrm{dt}=0$,

$$
\mathrm{d}\left[\mathrm{p}^{(1)}+\mathrm{q}_{\mathrm{s}}^{(1)}\right] / \mathrm{dt}=0
$$

confirming the conservation of polymer mass. It follows that

$$
\mathrm{dp}^{(1)} / \mathrm{dt}=-\mathrm{dq}_{\mathrm{s}}^{(1)} / \mathrm{dt}=-\mathrm{x}_{\mathrm{s}} \mathrm{dq}_{\mathrm{s}}^{(0)} / \mathrm{dt}
$$


When $\mathrm{k}_{\mathrm{as}}=0$,

$$
\mathrm{q}_{\mathrm{s}}^{(1)}(\mathrm{t})=\mathrm{k}_{\mathrm{s}} \mathrm{x}_{\mathrm{s}} \mathrm{p}_{0}^{(0)} \mathrm{t}
$$

and

$$
\mathrm{p}^{(1)}(\mathrm{t})=\mathrm{p}_{0}^{(0)}\left[1-\mathrm{k}_{\mathrm{S}} \mathrm{x}_{\mathrm{S}} \mathrm{t}\right]
$$

The mass concentration of polymer decreases linearly with time, so that total mass, $\mathrm{q}_{\mathrm{s}}^{(1)}+\mathrm{p}^{(1)}$, is constant. The time when all the polymer disappears to form the specific product, $\mathrm{x}_{\mathrm{S}}$, can also be calculated from the above equation.

The second moment equations are

$$
\begin{aligned}
\mathrm{dp}^{(2)} / \mathrm{dt} & =-\left(\mathrm{k}_{\mathrm{he}}+\mathrm{k}_{\mathrm{de}} \mathrm{C}\right) \mathrm{p}^{(2)}+\left(\mathrm{k}_{\mathrm{He}}+\mathrm{k}_{\mathrm{De}} \mathrm{C}\right) \mathrm{r}_{\mathrm{e}}{ }^{(2)} \\
\mathrm{dq}_{\mathrm{s}}{ }^{(2)} / \mathrm{dt} & =\mathrm{k}_{\mathrm{bs}} \mathrm{x}_{\mathrm{s}}{ }^{2} \mathrm{r}_{\mathrm{s}}^{(0)}-\mathrm{k}_{\mathrm{as}} \mathrm{q}_{\mathrm{s}}^{(2)} \mathrm{r}_{\mathrm{e}}^{(0)} \\
\mathrm{dr}_{\mathrm{e}}{ }^{(2)} / \mathrm{dt} & =\left(\mathrm{k}_{\mathrm{he}}+\mathrm{k}_{\mathrm{de}} \mathrm{C}\right) \mathrm{p}^{(2)}-\left(\mathrm{k}_{\mathrm{He}}+\mathrm{k}_{\mathrm{De}} \mathrm{C}\right) \mathrm{r}_{\mathrm{e}}^{(2)}-\mathrm{k}_{\mathrm{ih}} \mathrm{r}_{\mathrm{e}}^{(2)}+\mathrm{k}_{\mathrm{iH}} \mathrm{r}_{\mathrm{s}}^{(2)} \\
& +\mathrm{k}_{\mathrm{bs}}\left(\mathrm{x}_{\mathrm{s}}{ }^{2} \mathrm{r}_{\mathrm{s}}^{(0)}-2 \mathrm{x}_{\mathrm{s}} \mathrm{r}_{\mathrm{s}}^{(1)}+\mathrm{r}_{\mathrm{s}}{ }^{(2)}\right)-\mathrm{k}_{\mathrm{as}} \mathrm{r}_{\mathrm{e}}^{(2)} \mathrm{p}_{\mathrm{s}}^{(0)} \\
\mathrm{dr}_{\mathrm{s}}{ }^{(2)} / \mathrm{dt} & =\mathrm{k}_{\mathrm{ih}} \mathrm{r}_{\mathrm{e}}^{(2)}-\mathrm{k}_{\mathrm{iH}} \mathrm{r}_{\mathrm{s}}^{(2)}-\mathrm{k}_{\mathrm{bs}} \mathrm{r}_{\mathrm{s}}^{(2)} \\
+ & \mathrm{k}_{\mathrm{as}}\left(\mathrm{r}_{\mathrm{e}}{ }^{(2)} \mathrm{q}_{\mathrm{s}}^{(0)}+2 \mathrm{r}_{\mathrm{e}}^{(1)} \mathrm{q}_{\mathrm{s}}^{(1)}+\mathrm{r}_{\mathrm{e}}^{(0)} \mathrm{q}_{\mathrm{s}}^{(2)}\right)
\end{aligned}
$$

With $\mathrm{k}_{\mathrm{as}}=0$,

$$
\mathrm{q}_{\mathrm{s}}^{(2)}(\mathrm{t})=\mathrm{x}_{\mathrm{s}}^{2} \mathrm{q}_{\mathrm{s}}^{(0)}=\mathrm{k}_{\mathrm{s}} \mathrm{x}_{\mathrm{s}}^{2} \mathrm{p}_{0}^{(0)} \mathrm{t}
$$

and

$$
\begin{aligned}
& \mathrm{dp}^{(2)} / \mathrm{dt}=\mathrm{k}_{\mathrm{s}} \mathrm{x}_{\mathrm{s}}{ }^{2} \mathrm{p}_{0}{ }^{(0)}-2 \mathrm{k}_{\mathrm{s}} \mathrm{x}_{\mathrm{s}} \mathrm{p}^{(1)}+2 \mathrm{k}_{\mathrm{s}}{ }^{2} \mathrm{x}_{\mathrm{s}} \mathrm{p}_{0}{ }^{(0)} / \mathrm{k}_{\mathrm{he}} \\
& \mathrm{p}^{(2)}(\mathrm{t})=\mathrm{p}_{0}{ }^{(2)}+2 \mathrm{x}_{\mathrm{s}}{ }^{2} \mathrm{p}_{0}{ }^{(0)} \mathrm{k}_{\mathrm{s}} \mathrm{t}\left(1+\mathrm{k}_{\mathrm{s}} \mathrm{t}\right)-2 \mathrm{k}_{\mathrm{s}} \mathrm{t} \mathrm{x}_{\mathrm{s}} \mathrm{p}_{0}^{(1)}+2 \mathrm{k}_{\mathrm{s}}{ }^{2} \mathrm{t} \mathrm{x}_{\mathrm{s}} \mathrm{p}_{0}{ }^{(0)} / \mathrm{k}_{\mathrm{he}}
\end{aligned}
$$

Eqs 2.24, 2.32, 2.38 indicate that the specific product moments, $\mathrm{q}_{\mathrm{s}}{ }^{(\mathrm{n})}(\mathrm{t})$, increase linearly with time. This is consistent with the equation obtained by a simpler mechanism ignoring the radicals (Wang et al., 1995) and accurately describes the experimental data (Wang et al., 1995; Madras et al., 1995, 1996).

The equations obtained for the polymer moments, Eqs. 2.21, 2.33, 2.40, are identical to the equations derived with a simpler mechanism (McCoy and Madras, 1996) if terms involving $\mathrm{k}_{\mathrm{S}}{ }^{2}$ are ignored. The new mechanism provides more detailed information on the radicals, 
however, and also provides an explanation for the activation energies observed for random scission and chain-end scission (Kodera and McCoy, 1997).

\section{Results and Discussion}

The random-scission degradation rate coefficient, $\mathrm{k}_{\mathrm{r}}$, was determined from the experimental data by analyzing the time dependence of the polystyrene MWDs. Figure 1 shows $\mathrm{p}_{\text {tot }}{ }^{(0)} / \mathrm{p}_{\text {tot } 0}{ }^{(0)}$ plotted as a function of time for various hydrogen donor concentrations, where $\mathrm{p}_{\operatorname{tot} 0}(0)$ is the initial molar concentration of the polystyrene $(\mathrm{mol} / \mathrm{L})$ and $\mathrm{p}_{\mathrm{tot}}{ }^{(0)}$ is the molar concentration of the polystyrene $(\mathrm{mol} / \mathrm{L})$ as a function of time. The plots show a rapid increase in the zeroth moment (corresponding to a rapid decrease of MW) at times less than 45 minutes. This is attributed to scission of weak links (Stivala et al., 1983; Madras et al., 1996c) randomly distributed along the polymer chain (Chiantore et al., 1981).

We can assume that the weak links are totally depleted after 45 minutes. The initial molar concentration of the strong links in polystyrene, $\mathrm{p}_{0}{ }^{(0)}$, is determined from the intercept of the regressed line of the $\mathrm{p}_{\text {tot }}{ }^{(0)} / \mathrm{p}_{\text {tot } 0}{ }^{(0)}$ data for $\mathrm{t} \geq 45$ minutes. The slopes, corresponding to the rate coefficient for random scission, $\mathrm{k}_{\mathrm{r}}$, are determined from the plot of $\ln \left(\mathrm{p}^{(0)} / \mathrm{p}_{0}{ }^{(0)}\right)$ versus time (Figure 2). The rate coefficient decreases with increasing hydrogen donor (6-hydroxy tetralin) concentration (Figure 3). This behavior is consistent with eq. 1.21 when $\mathrm{k}_{\mathrm{d}}=0$ (Figure 4). Because $\mathrm{k}_{\mathrm{D}} \neq 0$, radicals are quenched by the hydrogen donor according to eq. 1.4a.

The rate coefficient for random chain scission depends on hydrogen donor concentration through the fundamental radical rate parameters, $\mathrm{k}_{\mathrm{b}}, \mathrm{k}_{\mathrm{h}}, \mathrm{k}_{\mathrm{d}}, \mathrm{k}_{\mathrm{H}}, \mathrm{k}_{\mathrm{D}}$ (eq. 1.21). Figure 4 shows the different effects of the hydrogen donor solvent on the degradation rate coefficient. When $\mathrm{k}_{\mathrm{h}}=0$, the rate coefficient has a first-order dependence at low hydrogen donor concentrations and a zero-order dependence at high hydrogen donor concentrations. This has been experimentally observed (Figure 5) for poly(styrene-allyl alcohol) degradation in the presence of tetralin at $150^{\mathrm{c}}$ $\mathrm{C}$ (Madras et al., 1995). When $\mathrm{k}_{\mathrm{H}}=0$, the rate coefficient has a negative order dependence, as observed for the polystyrene degradation in the presence of hydrogen donors like tetralin (Sato et 
al., 1991) and 6-hydroxy tetralin (Figure 3). When $\mathrm{k}_{\mathrm{d}}=\mathrm{k}_{\mathrm{D}}=0$, the rate coefficient is independent of the hydrogen donor concentration. This is consistent with poly( $\alpha$-methyl styrene) degradation in the presence of hydrogen donor solvents. For chain-end scission, the effect of hydrogen donor concentration is qualitatively similar to that in Figure 4.

The chain-end scission of polystyrene yields specific products of styrene and various oligomers. The production rates of these specific products, however, could not be determined because the 6-hydroxy tetralin overlapped the monomer and oligomer peaks. We examined instead the chain-end scission rates for the degradation of poly(styrene allyl-alcohol) in the presence of tetralin at $150{ }^{\circ} \mathrm{C}$ (Madras et al., 1995). The specific product molar concentrations are linear in time, as predicted by eq. 2.23. The rate coefficient for chain-end scission, $\mathrm{k}_{\mathrm{s}}$, can be obtained from the slope of the molar concentration of the specific product versus time. Figure 6 depicts the influence of tetralin on the rate of chain-end scission of poly(styrene allyl-alcohol) to an oligomer of styrene and allyl alcohol (SA) at $150{ }^{\circ} \mathrm{C}$ (Madras et al., 1995). The dependence is consistent with eq. 2.24 (for $\mathrm{k}_{\mathrm{he}}=0$ ), which relates the chain-end scission rate coefficient to the hydrogen donor concentration.

\section{Concluding Remarks}

A macromolecule typically fragments into smaller chains by random scission and to a lesser extent into oligomers and monomers by chain-end scission. The proposed model for chain scission degradation reactions accounts for the radical mechanisms of initiation-termination, hydrogen abstraction, degradation and repolymerization reactions. The theoretical analysis, based on the continuous distribution kinetics of homologous macromolecules, explains the different effects of hydrogen donors on polymer degradation. The effect of hydrogen donor concentration, $\mathrm{C}$, on the random and chain-end scission rate coefficients is given by eqs. 1.21 and 2.24 , respectively.

\section{Acknowledgment}

The financial support of Pittsburgh Energy Technology Center Grant No. DOE DEFG22-94PC94204 and EPA Grant No. CR 822990-01-0 is gratefully acknowledged. The authors thank Professor J.M. Smith and Dr. Yoichi Kodera for helpful discussions. 


\section{References}

Angelova, G.; Kamenski, D.; Dimova, N. "Kinetics of Donor-Solvent Liquefaction of Bulgarian Brown Coal.," Fuel, 68, 1434 (1989).

Chawla, B.; Keogh, R.; Davis, B.H.; "Effect of Coal Properties, Temperature, and Mineral Matter upon a Hydrogen-donor Solvent during Coal Liquefaction," Energy and Fuels, 3, 236 (1989).

Cameron, G.G., J.R. MacCallum, "The Thermal Degradation of Polystyrene," J. Macromol. Sci., Rev. Macromol. Chem., C1(2), 327 (1967).

Cameron, G.G. ; Kerr, G. P. "Thermal Degradation of Polystyrene -I," Europ. Polym. J., 4, 709 (1968).

Chiantore, O.; Camino, G.; Costa, L.; Grassie, N., "Weak Links in Polystyrene," Poly. Deg. and Stab., 3, 209 (1981).

Chiba, K.; Tagaya, H.; Yamauchi, T.; Sato, S., "Effects of Solvents on Thermal Cracking of Model Compounds Typical of Coal," I\&EC Research, 30, 1141 (1991).

Clough, R.L.; Billingham, N.C.; Gillen, K.T., Polymer Durability, ACS Symposium Series (1996).

Flynn, J.H.; Florin, R.E. Degradation and Pyrolysis Mechanisms In Pyrolysis and GC in Polymer Analysis, Liebman, S. A.; Levy, E.J., Eds., Marcel Dekker Inc., New York, 1985, p. 149.

Jellinek, H.H.G., "Degradation of Polystyrene," J. Polym. Sci., 3, 850 (1948).

Kodera, Y. ; B.J. McCoy, "Continuous-Distribution Kinetics of Radical Mechanisms for Polymer Decomposition and Repolymerization," AIChE J., (1997); In Review.

Madras, G., J.M. Smith, B.J. McCoy, "Effect of Tetralin on the Degradation of Polymer in Solution," I\&EC Research, 34, 4222 (1995).

Madras, G., J.M. Smith, B.J. McCoy, "Thermal Degradation of Poly( $\alpha$-Methylstyrene) in Solution," Poly. Deg. and Stab., 52, 349 (1996a).

Madras, G., J.M. Smith, B.J. McCoy, "Degradation of Poly(Methyl Methacrylate) in Solution," I\&EC Research, 35, 1795 (1996b).

Madras, G., J.M. Smith, B.J. McCoy, "Thermal Degradation Kinetics of Polystyrene in Solution," Poly. Deg. and Stab., (1996c); In press.

Madras, G., B.J. McCoy, "Oxidative Degradation Kinetics of Polystyrene in Solution," Chem. Eng. Sci., (1996); In review.

McCaffrey, W.C., Brues, M.J.; Cooper, D.G.; Kamal, M.R., "Thermolysis of Polyethylene Polystyrene Mixtures," J. App. Poly. Sci., 60, 2133 (1996).

McCoy, B.J., G. Madras, "Degradation Kinetics of Polymers in Solution: Dynamics of Molecular Weight Distributions," AIChE J., (1996); In press. 
94204R10.PDF

April 16, 1997

McCoy, B.J., M. Wang, "Continuous-mixture fragmentation kinetics: Particle size reduction and molecular cracking," Chem. Eng. Sci., 49, 3773 (1994).

McNeill, I.C.; Zulfiqar, M.; Kousar, T. "A Detailed Investigation of the Products of the Thermal Degradation of Polystyrene," Poly. Degr. and Stab., 28, 131 (1990).

Miller, A. "Industry Invests in Reusing Plastics," Env. Sci. Tech., 28, 16A (1994).

Murakata, T.; Saito, Y.; Yosikawa, T.; Suzuki, T.; Sato, S. "Solvent Effect on Thermal Degradation of Polystrene and Poly- $\alpha$-methylstyrene," Polymer, 34, 1436 (1993).

Murakata, T.; Wagatsuma, S..; Saito, Y.; Suzuki, T.; Sato, S. "Effect of Solvent on Thermal Degradation of Poly (para-methylstyrene)," Polymer, 34, 1431 (1993).

Nigam, A; Fake, D. M; Klein M.T. "Simple Approximate Rate Law for Both Short-Chain and Long Chain Rice Herzfeld Kinetics," AIChE J., 40, 908 (1994).

Reich, L.; Stivala, S.S. Elements of Polymer Degradation, McGraw-Hill, New York, 1971, p. 186.

Mochida, I.; Takayama, A.; Sakata, R.; Sakanishi, K. "Hydrogen-Transferring Liquefaction of an Australian Brown Coal with Polyhydrogenated Condensed Aromatics - Roles of Donor in the Liquefaction," Energy and Fuels, 4, 81 (1990).

Sato, S.; Murakata, T.; Baba, S.; Saito, Y.; Watanabe, S. "Solvent Effect on Thermal Degradation of Polystyrene," J. Appl. Poly. Sci., 40, 2065 (1990).

Song, C.; Lai, W.C.; Schobert, H.H. "Hydrogen-Transferring Pyrolysis of Long-Chain Alkanes and Thermal Stability Improvement of Jet Fuels by Hydrogen Donors." I\&EC Research, 33, 548 (1994).

Stivala, S.S.; Kimura, J.; Reich, L. "The Kinetics of Degradation Reactions," In Degradation and Stabilization of Polymers, H.H.G. Jellinek, ed., Elsevier, p. 1-66. (1983).

Vollhardt, K.P.C. ; Schore, N.E. In Organic Chemistry, 2nd Ed., Freeman (1994); p 111.

Wang, M., J.M. Smith, B.J. McCoy, "Continuous Kinetics for Thermal Degradation of Polymer in Solution," AIChE J., 41, 1521 (1995).

Wang, M., C. Zhang, J.M. Smith, B.J. McCoy, "Continuous-Mixture Kinetics of Thermolytic Extraction of Coal in Supercritical Fluid," AIChE J., 40, 131 (1994).

Wang, M., J.M. Smith, B.J. McCoy, "Kinetics of Coal Thermolysis with a Mixed Solvent of Tert-butyl Alcohol and Tetralin," Energy \& Fuels, 7, 78 (1993). 


\section{Figure captions}

1. Plot of $\mathrm{p}_{\text {tot }}{ }^{(0)} / \mathrm{p}_{\mathrm{tot} 0}{ }^{(0)}$ versus time for polystyrene degradation at $275^{\mathrm{c}} \mathrm{C}$ for four hydrogen donor (6-hydroxy tetralin) concentrations.

2. Plot of $\ln \left(\mathrm{p}^{(0)} / \mathrm{p}_{0}{ }^{(0)}\right)$ versus time for polystyrene degradation at $275{ }^{\circ} \mathrm{C}$ for four hydrogen donor (6-hydroxy tetralin) concentrations, to determine the linearly regressed slopes, $\mathrm{k}_{\mathrm{r}}$ (eq. 1.21).

3. Effect of hydrogen donor (6-hydroxy tetralin) mass concentration, $\mathrm{C}_{\mathrm{m}}$, on the rate coefficient of random chain scission, $\mathrm{k}_{\mathrm{r}}$, of polystyrene at $275^{\circ} \mathrm{C}$.

4. Plot of the rate coefficient of random chain scission, $\mathrm{k}_{\mathrm{r}}$, versus hydrogen donor concentration (mol/vol), C, based on eq. 1.21 to show the different effects of the hydrogen donor concentration and rate parameters.

5. Effect of hydrogen donor (tetralin) concentration (in vol\%), $\mathrm{C}_{\mathrm{v}}$, on the rate coefficient of random chain scission, $\mathrm{k}_{\mathrm{r}}$, of poly(styrene allyl-alcohol) at $150{ }^{\circ} \mathrm{C}$.

6. Effect of hydrogen donor (tetralin) concentration (in vol\%), $\mathrm{C}_{\mathrm{v}}$, on the rate coefficient of chain-end scission, $\mathrm{k}_{\mathrm{s}}$, of poly(styrene allyl-alcohol) to SA at $150{ }^{\circ} \mathrm{C}$.

Legend for figures 1,2

Polystyrene (2 g/L)

- Polystyrene $(2 \mathrm{~g} / \mathrm{L})+6$-hydroxy tetralin $(2 \mathrm{~g} / \mathrm{L})$

Polystyrene $(2 \mathrm{~g} / \mathrm{L})+6$-hydroxy tetralin $(5 \mathrm{~g} / \mathrm{L})$

Polystyrene $(2 \mathrm{~g} / \mathrm{L})+6$-hydroxy tetralin $(10 \mathrm{~g} / \mathrm{L})$ 
Figure 1. Plot of $\mathrm{p}_{\text {tot }}{ }^{(0)} / \mathrm{p}_{\text {tot } 0}{ }^{(0)}$ versus time for polystyrene degradation at $275{ }^{\circ} \mathrm{C}$ for four hydrogen donor (6-hydroxy tetralin) concentrations.

Polystyrene $(2 \mathrm{~g} / \mathrm{L})$

$\checkmark$ Polystyrene $(2 \mathrm{~g} / \mathrm{L})+6$-hydroxy tetralin $(2 \mathrm{~g} / \mathrm{L})$

Polystyrene $(2 \mathrm{~g} / \mathrm{L})+6$-hydroxy tetralin $(5 \mathrm{~g} / \mathrm{L})$

Polystyrene $(2 \mathrm{~g} / \mathrm{L})+6$-hydroxy tetralin $(10 \mathrm{~g} / \mathrm{L})$

Figure 2. Plot of $\ln \left(\mathrm{p}^{(0)} / \mathrm{p}_{0}{ }^{(0)}\right)$ versus time for polystyrene degradation at $275{ }^{\circ} \mathrm{C}$ for four hydrogen donor (6-hydroxy tetralin) concentrations, to determine the linearly regressed slopes, $\mathrm{k}_{\mathrm{r}}$ (eq. 1.21).

Polystyrene $(2 \mathrm{~g} / \mathrm{L})$

- Polystyrene $(2 \mathrm{~g} / \mathrm{L})+6$-hydroxy tetralin $(2 \mathrm{~g} / \mathrm{L})$

Polystyrene $(2 \mathrm{~g} / \mathrm{L})+6$-hydroxy tetralin $(5 \mathrm{~g} / \mathrm{L})$

Polystyrene $(2 \mathrm{~g} / \mathrm{L})+6$-hydroxy tetralin $(10 \mathrm{~g} / \mathrm{L})$

Figure 3. Effect of hydrogen donor (6-hydroxy tetralin) mass concentration, $\mathrm{C}_{\mathrm{m}}$, on the rate coefficient of random chain scission, $\mathrm{k}_{\mathrm{r}}$, of polystyrene at $275^{\circ} \mathrm{C}$.

Figure 4. Plot of the rate coefficient of random chain scission, $\mathrm{k}_{\mathrm{r}}$, versus hydrogen donor concentration $(\mathrm{mol} / \mathrm{vol}), \mathrm{C}$, based on eq. 1.21 to show the different effects of the hydrogen donor concentration and rate parameters.

Figure 5. Effect of hydrogen donor (tetralin) concentration (in vol\%), $\mathrm{C}_{\mathrm{v}}$, on the rate coefficient of random chain scission, $\mathrm{k}_{\mathrm{r}}$, of poly(styrene allyl-alcohol) at $150{ }^{\circ} \mathrm{C}$.

Figure 6. Effect of hydrogen donor (tetralin) concentration (in vol\%), $\mathrm{C}_{\mathrm{v}}$, on the rate coefficient of chain-end scission, $\mathrm{k}_{\mathrm{S}}$, of poly(styrene allyl-alcohol) to SA at $150{ }^{\circ} \mathrm{C}$. 


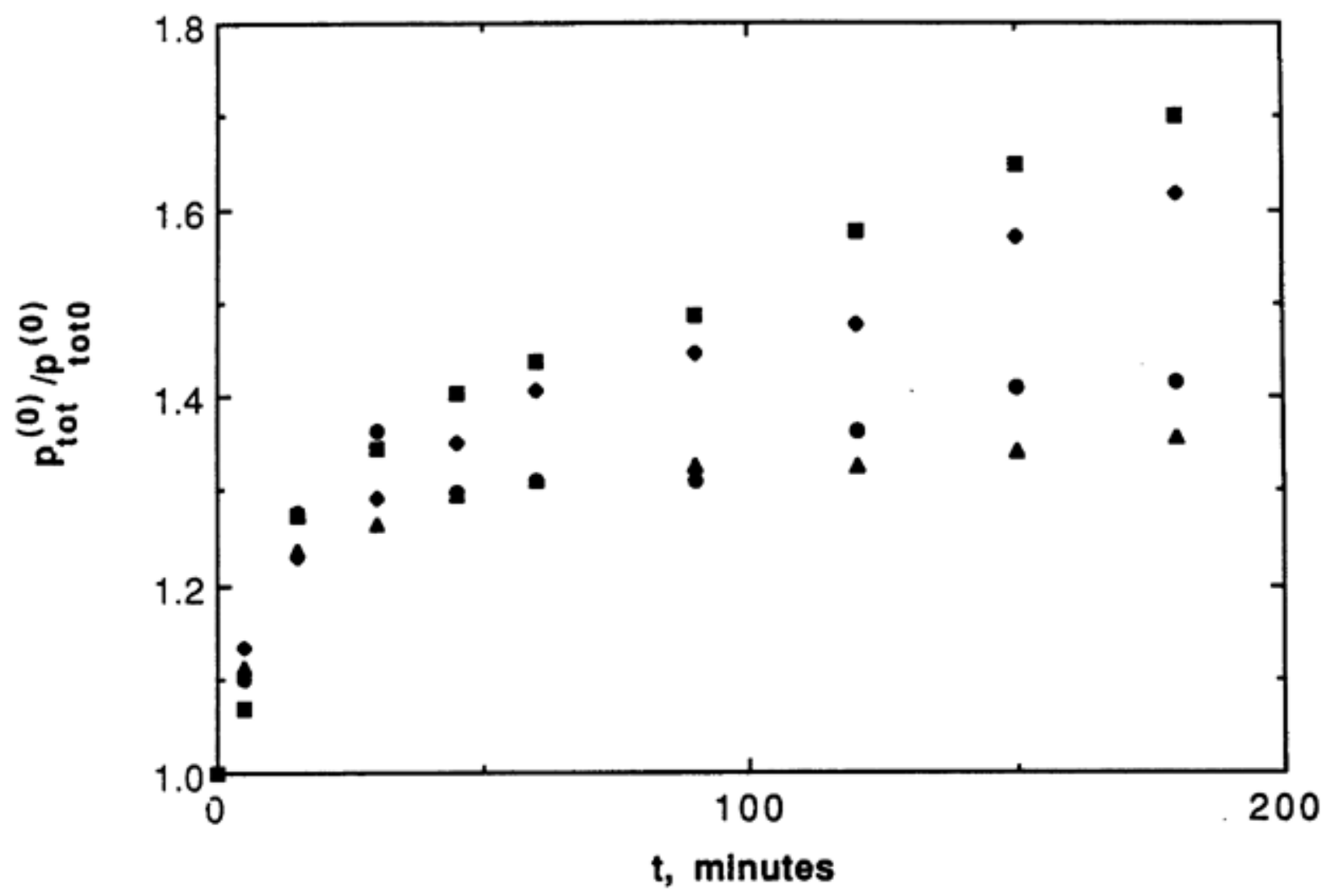

Figure 1. Plot of $\mathrm{p}_{\mathrm{Lox}}{ }^{(0)} / \mathrm{p}_{\mathrm{Lot} 0}{ }^{(0)}$ versus time for polystyrene degradation at $275^{\circ} \mathrm{C}$ for four hydrogen donor (6-hydroxy tetralin) concentrations.

Polystyrene (2 g/L)

- Polystyrene $(2 \mathrm{~g} / \mathrm{L})+6$-hydroxy tetralin $(2 \mathrm{~g} / \mathrm{L})$

- Polystyrene $(2 \mathrm{~g} / \mathrm{L})+6$-hydroxy tetralin $(5 \mathrm{~g} / \mathrm{L})$

$\Delta$ Polystyrene $(2 \mathrm{~g} / \mathrm{L})+6$-hydroxy tetralin $(10 \mathrm{~g} / \mathrm{L})$ 


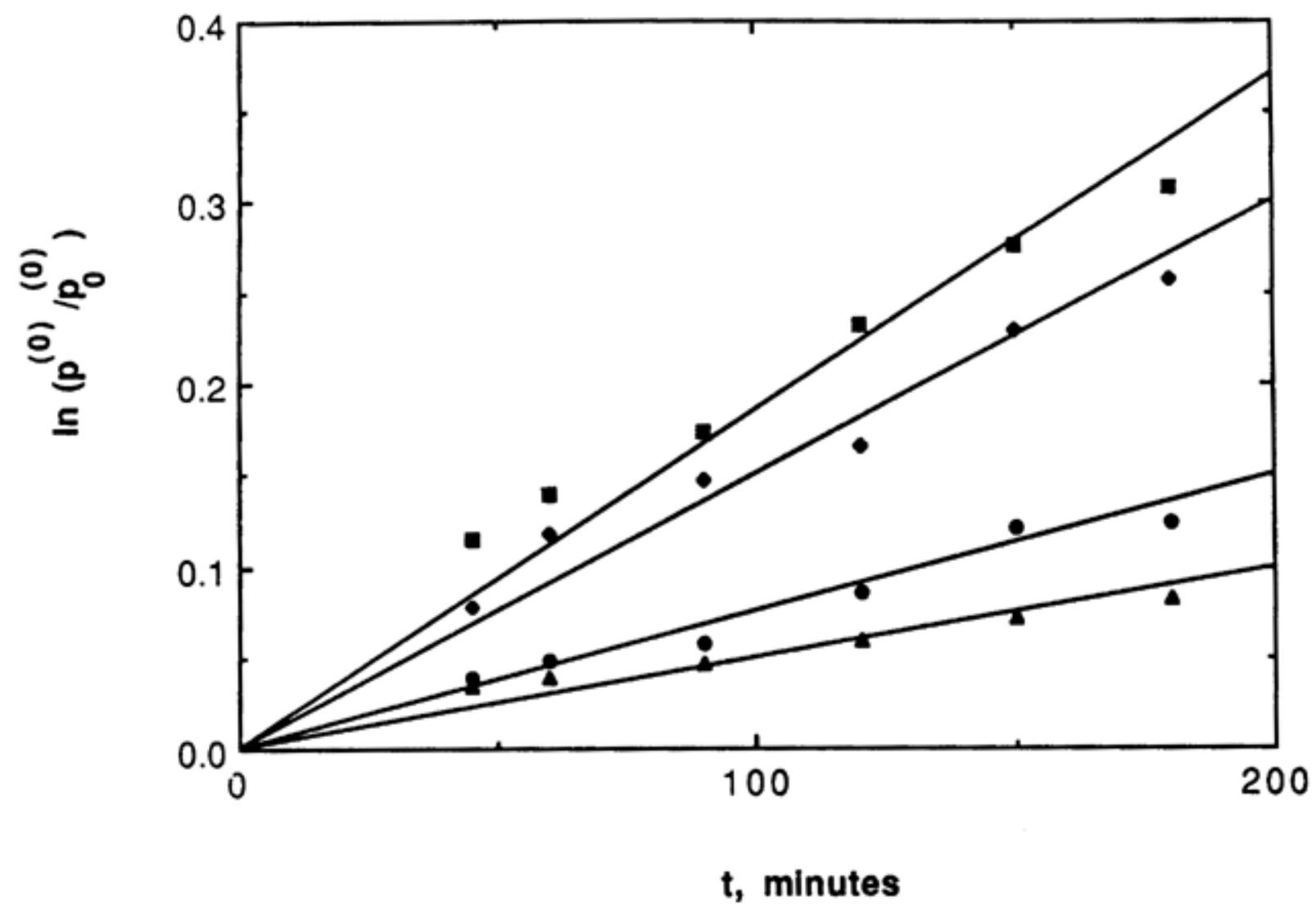

Figure 2. Plot of $\ln \left(\mathrm{p}^{(0)} / \mathrm{p}_{0}{ }^{(0)}\right)$ versus time for polystyrene degradation at $275^{\circ} \mathrm{C}$ for four hydrogen donor (6-hydroxy tetralin) concentrations, to determine the linearly regressed slopes, $\mathrm{k}_{\mathrm{r}}$ (eq. 1.21).

Polystyrene ( $2 \mathrm{~g} / \mathrm{L})$

- Polystyrene $(2 \mathrm{~g} / \mathrm{L})+6$-hydroxy tetralin $(2 \mathrm{~g} / \mathrm{L})$

- Polystyrene $(2 \mathrm{~g} / \mathrm{L})+6$-hydroxy tetralin $(5 \mathrm{~g} / \mathrm{L})$

$\Delta$ Polystyrene $(2 \mathrm{~g} / \mathrm{L})+6$-hydroxy tetralin $(10 \mathrm{~g} / \mathrm{L})$ 


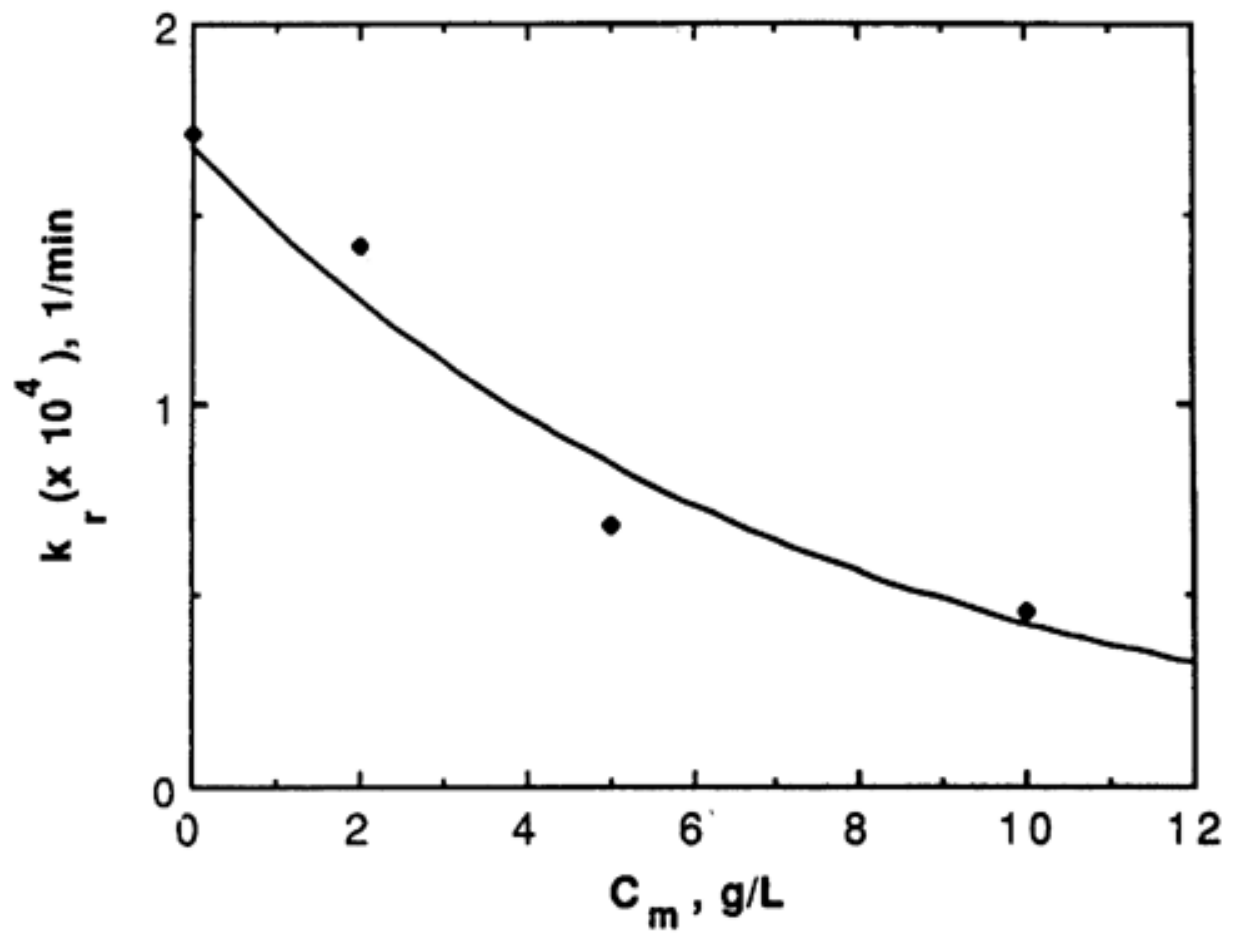

Figure 3. Effect of hydrogen donor (6-hydroxy tetralin) mass concentration, $C_{m}$, on the rate coefficient of random chain scission, $\mathrm{k}_{\mathrm{r}}$, of polystyrene at $275^{\circ} \mathrm{C}$. 


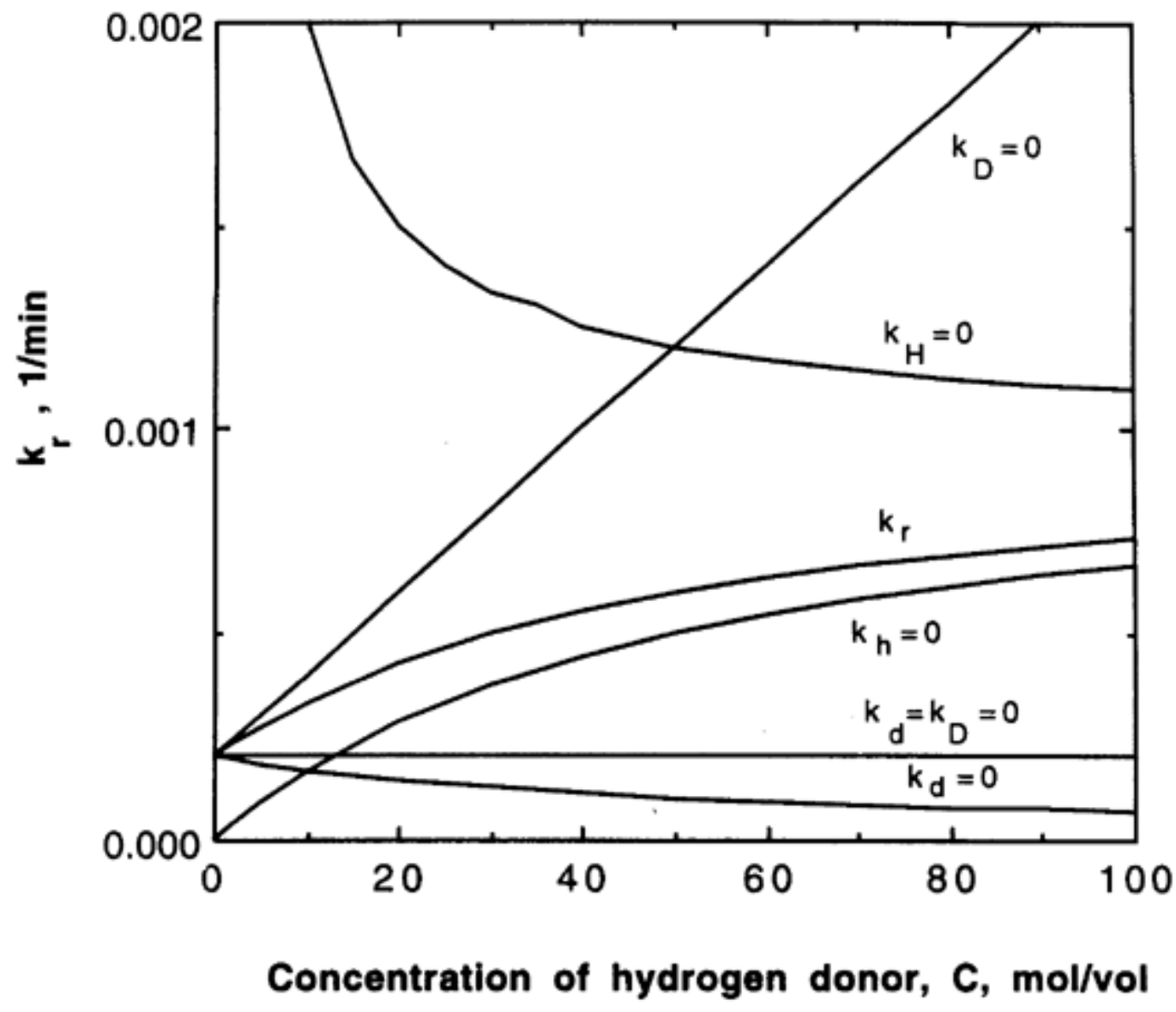

Figure 4. Plot of the rate coefficient of random chain scission, $k_{r}$, versus hydrogen donor concentration (mol/vol), C, based on eq. 1.21 to show the different effects of the hydrogen donor concentration and rate parameters. 


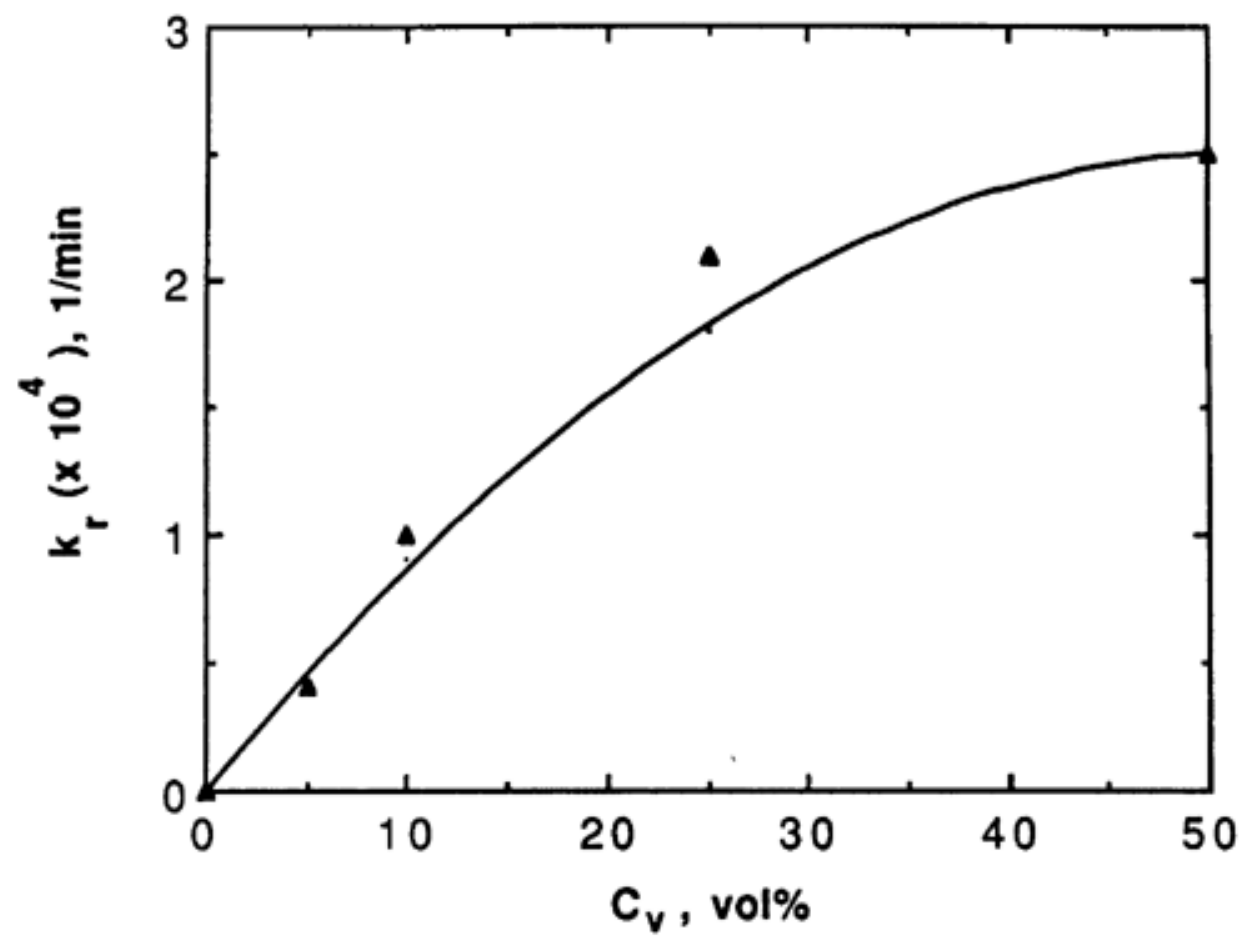

Figure 5. Effect of hydrogen donor (tetralin) concentration (in vol\%), $\mathrm{C}_{\mathrm{v}}$, on the rate coefficient of random chain scission, $\mathrm{k}_{\mathrm{r}}$, of poly(styrene allyl-alcohol) at $150^{\circ} \mathrm{C}$. 


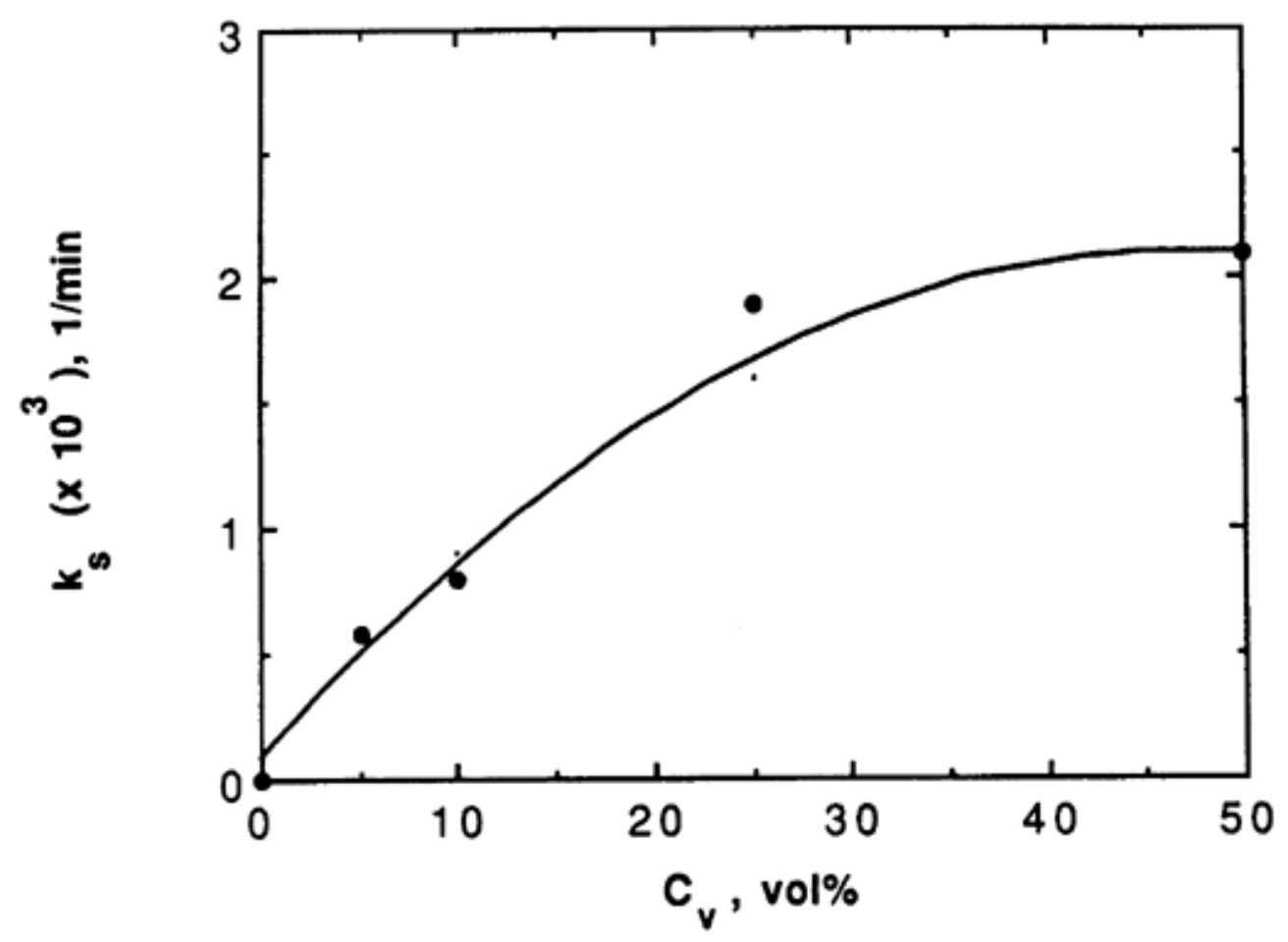

Figure 6. Effect of hydrogen donor (tetralin) concentration (in vol\%), $\mathrm{C}_{\mathrm{v}}$, on the rate coefficient of chain-end scission, $k_{s}$, of poly(styrene allyl-alcohol) to $\mathrm{SA}$ at $150^{\circ} \mathrm{C}$. 